\title{
THE 3D SCANNING IMAGE QUALITY OF THE INTERIOR WITH THE MIRROR SURFACE
}

\author{
Irena Drofova, Milan Adamek \& Vaclav Mach
}
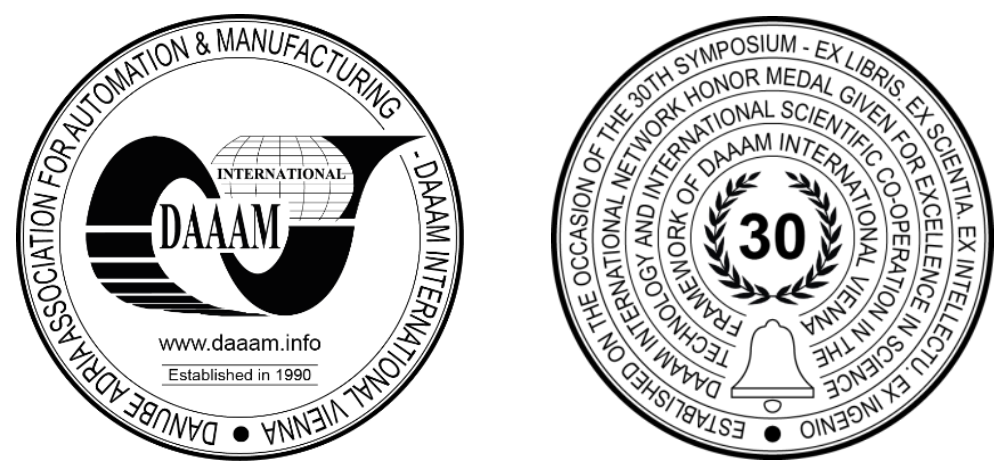

This Publication has to be referred as: Drofova, I[rena]; Adamek, M[ilan] \& Mach, V[aclav] (2020). The 3D Scanning Image Quality of the Interior with the Mirror Surface, Proceedings of the 31st DAAAM International Symposium, pp.0826-0830, B. Katalinic (Ed.), Published by DAAAM International, ISBN 978-3-902734-29-7, ISSN 1726-9679, Vienna, Austria

DOI: $10.2507 / 31$ st.daaam.proceedings. 115

\begin{abstract}
This article deals with the theory of digital image transmission. Image quality and possible deterioration when scanning the interior using a 3D scanner. Noise in white areas has reduced image quality. Brightness and contrast values have been changed to increase quality. This adjustment suppressed noise, but not significantly. The mirror placed in the interior created a secondary 3D model and point clouds of the scanned space. The occlusion of point clouds from both 3D models could significantly increase image quality, especially in detail.
\end{abstract}

Keywords: brightness, contrast, noise, 3D scanner, photogrammetry

\section{Introduction}

The progressive rise of image digital technology and virtual reality environment great emphasis on the quality of graphic data processing for application in virtual environments. Data created in a graphical method and by the process of photogrammetry or 3D scanning technology of the real environment, always carry with them a great emphasis on the process of data acquisition. Also, for their transmission and data compression too.

Some rules need to be respected during the initial process of obtaining data as well as during their transfer and subsequent processing. In all the above methods of creating a digital image, the procedure of image transmission is created so that in each case the reality is simulated as best as possible in the highest possible quality, which is closest to the perception of the image by the human eye in the real environment. Here is the key choice of the device that best brings the created image to the human eye. This article deals with image degradation and modification. It discusses the criteria of brightness, which is considered a key variable as the selected image value and contrast for image preprocessing. [1] The color, the light spectrum, and color perception by the human eye with an emphasis on the quality of detail. One possibility is the inclusion of point clouds by a combination of 3D scanning and photogrammetry methods. Especially where low values of image resolution can be expected depending on the depth of the object from the background perceived by the observer. [2] Graphic correction of image brightness and contrast criteria for better image quality in detail can significantly affect image quality right at the beginning of the process. [3] The paper deals with the method of obtaining a 3D image by scanning the interior with a 3D laser scanner with a vaulted ceiling and a mirror. The no-traditional space of the room showed the main problem of the mentioned 3D scanning method. 


\subsection{Degradation of a digital image}

Often the only global information about a transferred image is a histogram, which gives us an idea of the brightness levels in a digital image. The value of each component corresponds to the number of points of the corresponding brightness in the image. We can use it when setting the conditions for scanning and digitalization, when changing the brightness scale, and when segmenting the image into objects and surroundings. When capturing, transmitting, processing, and displaying, the image is very often visibly degraded. We are therefore interested in image quality. The classic method is to evaluate the resolution. Describes the resolution of small and close objects. The image may have various alternative distortions, usually called noise. Noise can occur during image capture, transmission, and subsequent processing. It also causes disturbances in binary images, in which, for example, white pixels indicate objects and black ones indicate the background. Due to the noise, there is a unique black body in the area of the object and vice versa. We remove noise by smoothing the image. This method performs such noise filtering and suppresses higher frequencies of image functions. As an example of the consequence, this entails the suppression of desirable subsequent sudden changes, such as sharp lines and edges. [3]

Noise is mainly reflected in outdoor shooting under natural lighting. When shooting large areas, the quality of the display of details or smaller objects is low. The image quality is also affected by the weather and the degree of cloudiness when shooting. Shadows and sharp edges of objects also have a significant effect. To reduce the degree of image degradation due to noise the image smoothing method is often used.

\subsection{Enhance the brightness and contrast of the digital image}

The most common value expression of an image function is the brightness. Brightness is the quantity that best expresses the properties of an image signal in a way that corresponds to the perception of the image by the human eye. This value quantity is chosen mainly because it can be measured by almost all sensors for image input. Brightness is also an adjustable parameter on all devices that edit, capture, or reproduce an image. An indisputably important expression of brightness, as a basic value of the image function, is the fact that we thus avoid the complex creation of the image for the point of view of the description of the optical process. [3] Brightness values can affect the subsequent processing of a digital image. Especially, when the quality of the transmitted image and the image function differ depending on the type of lighting in the surroundings. Often when shooting in daylight, the image is qualitatively lower. The result is image degradation and exposed noise.

Enhance the image can also by adjusting the contrast values. Improving image contrast has found applications in many areas. For example, in the field of satellite imaging systems for better visibility of functions. A study and a comprehensive overview of various techniques for improving contrast were performed. This survey presents an increase in image quality through contrast while maintaining brightness values. [1]

\subsection{Image enhancement of point cloud occlusion}

In 2015 was the research-proven for high-quality 3D virtual visualization of a historic building. [2] 3D models of the historic building were created for documents and virtual realizations. 3D laser scanning and photogrammetry methods have been developed to integrate point clouds from scanned data. The choice of method depends on the scale and shape of the object and also on the applicability of the methods. Highly expensive devices such as 3D laser scanners collect spatial data. In contrast, cameras are inexpensive devices and are suitable not only for spatial imaging but also for capturing higher measurement details. The combination of two scanning methods proves to be very suitable for documenting a space with more complex details. In both cases, it is a matter of creating point clouds. [2]

In its simplest form, 3D data can be represented in a point cloud. Point clouds are a set of 3D data points, where each point is represented by three coordinates in the Cartesian system. Additional descriptions may provide information on colours and intensity. Point clouds, in general, contain only implicit neighbour relationships, so they are considered unstructured data. Point cloud segmentation is, to jeopardize explicit neighbourly relationships, a more complex field of research. [4] The import of external and internal parameters, the orientation of the scanning device, and its accuracy, provide data that can be read together with the images and will be used as initial information for the 3D reconstruction of the object. After loading this data, it is possible to build a point cloud. This step involves the main procedures of detecting and comparing information, and the result is a cloud of sparse points, resp. 3D representation of binding points of generated data in Agisoft Photoscan (Metashape) and point cloud view. [5] This software was also used in the study mentioned above.

\section{Type of 3D scanner for experimental shooting image}

The Leica BLK360 laser 3D scanner is a simple and efficient device for use in many fields. The easy handling and scanning speed of the device is an advantage for immediate $3 \mathrm{D}$ imaging. The scanning range is $360^{\circ} \mathrm{x} 300^{\circ}$. The scanner provides data up to a distance of $60 \mathrm{~m}$. [6] 


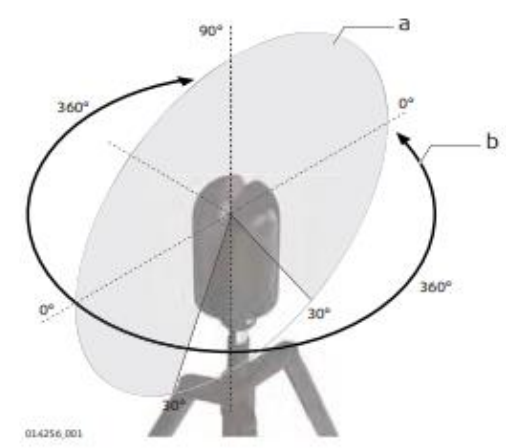

Fig. 1. The scanning range is $360^{\circ} \times 300^{\circ}$ [7]

The data registered by multiple applications and the data can be exported to many output formats to further image processing. Individual clouds can be combined into the coordinate system. The scanner works in a full-color panoramic image and two modes with a precise point cloud. [6] This scanner can collect two types of image data. The first is a panoramic $360^{\circ}$ spherical HDR image with three calibrated cameras and the second is a thermographic image with an infrared camera. [7] The scanning speed is 360000 dots per second. [8]
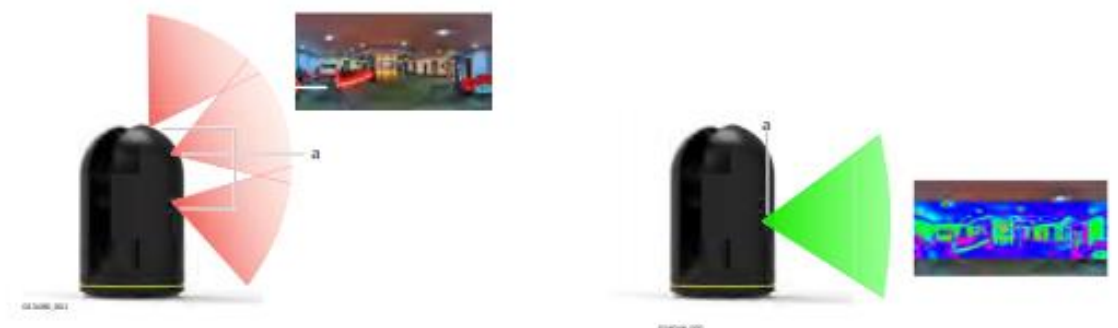

Fig. 2. Type of a panoramic $360^{\circ}$ image and a thermographic image [7]

Image data are transferring from the scanner in RAW format. That means this data are uncompressed in its original size. Such large data are demanding for further processing. This date needs to compress these large images data into another format. The choice of loss or lossless compression of image data depends on further processing and the purpose of using.

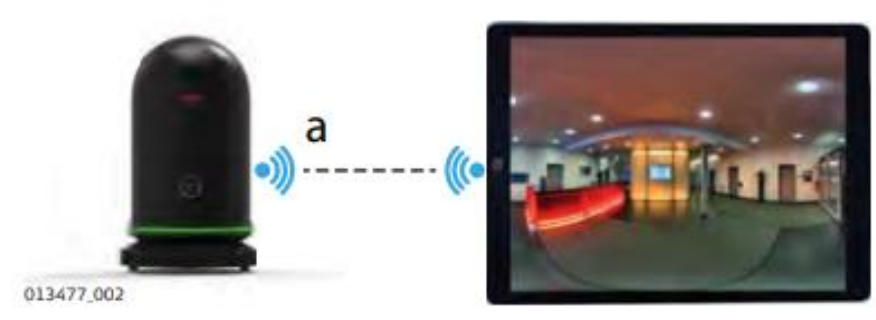

Fig. 3. Raw data transfer from BLK360 to computing device [7]

\section{Experimental 3D scanning an interior with the mirror}

An interior with the mirror was chosen to determine the level of image quality use by 3D scanning. This place has a vaulted ceiling and white walls. The dominant feature of the interior is a large mirror place between the windows in the room. The room was scanned in natural daylight. The interior was scanned from twelve positions, and more images were obtained from many angles.

\subsection{Image noise degrades the image quality}

The vaulted ceiling and many edges on the walls created many shadows in the daylight. The white color of the walls further affected the places where the high level of noise in the image manifested itself. These factors degraded the image directly on the input data. The positions of the scanner and the quality of the image are presents in the following figure. 

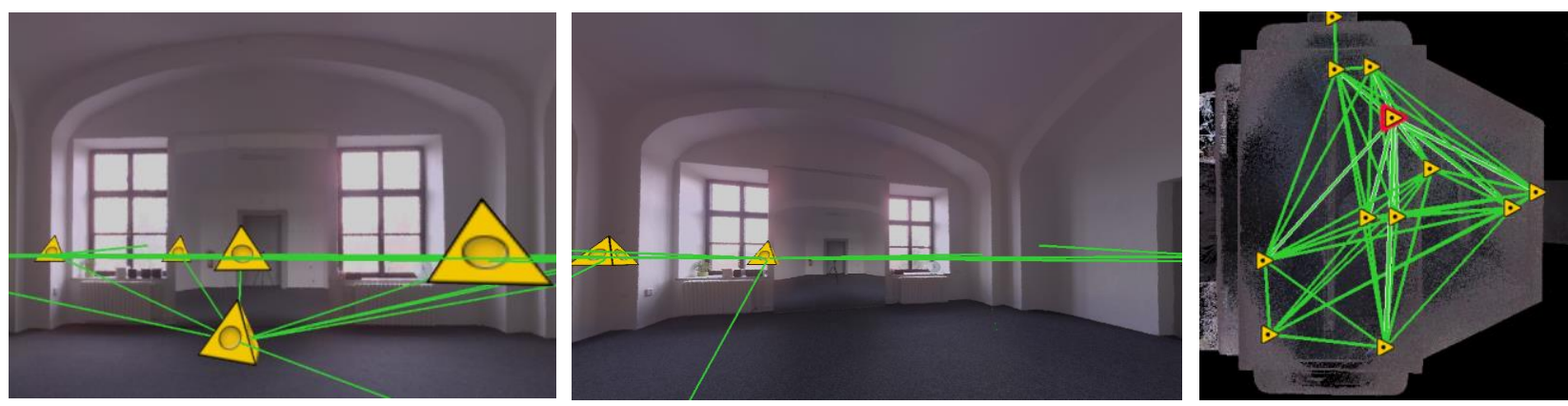

Fig. 4. The quality of the images and the positions of the scanner in the interior

In places where there is the most shadow, the noise manifested itself highly. Image smoothing can improve image quality. Before that, it is necessary to prepare the image and increase the image quality before the smoothing method. Brightness and contrast and maximum adjustment options are adjusted experimentally.

\subsection{Experimental adjusting the brightness and contrast of the image}

The basic adjustment of brightness and contrast was made in the following figures. The aim was to find out how to increase the quality of the scanned image in the RAW format before the next processing.

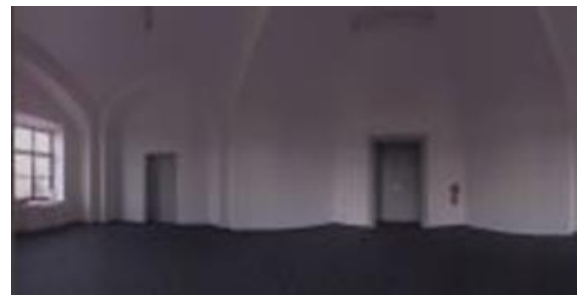

Fig. 5. The quality of original image

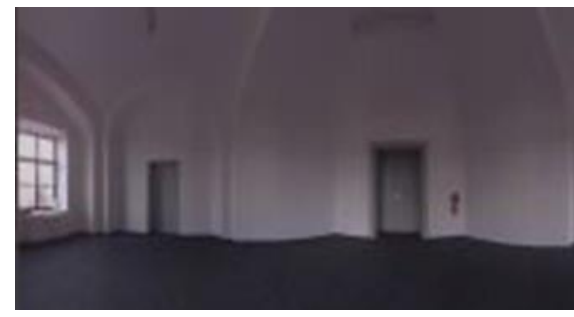

Fig. 7. The quality of original image

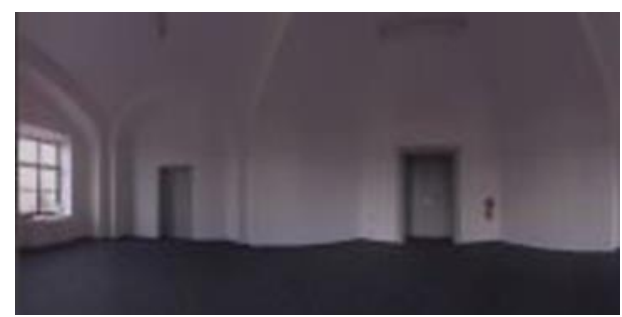

Fig. 9. The quality of original image

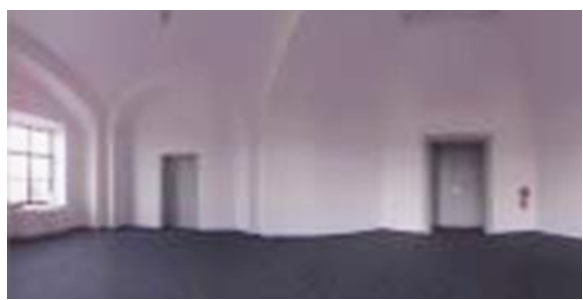

Fig. 6. Increase the brightness value $+90 \%$

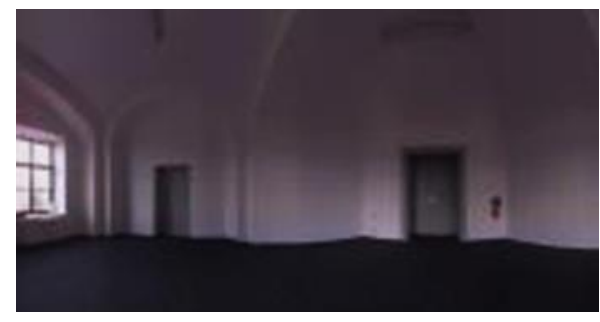

Fig. 8. Increase the contrast value $+90 \%$

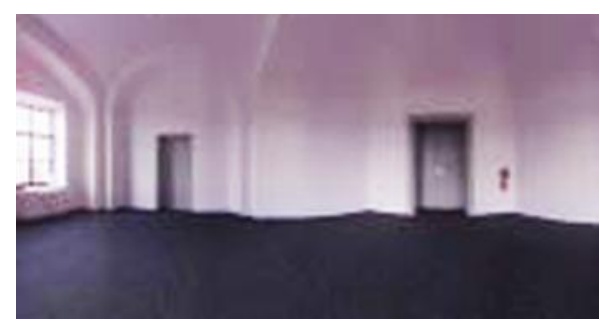

Fig. 10. Increase the brightness and contrast $+90 \%$

\subsection{Experimental scanning interior with a mirror}

A 3D scan of the space with a large mirror area on one of the walls created an inverse image. This image duplicates the scanned space. This also increased the total number of dots in the point cloud of the complete scanned image. The total point cloud contains 52164010 points. These points, in addition to the position, can describe the intensity, reflectance, and color information. The inverse image and the point cloud can be seen in figures 11 and 12 . 


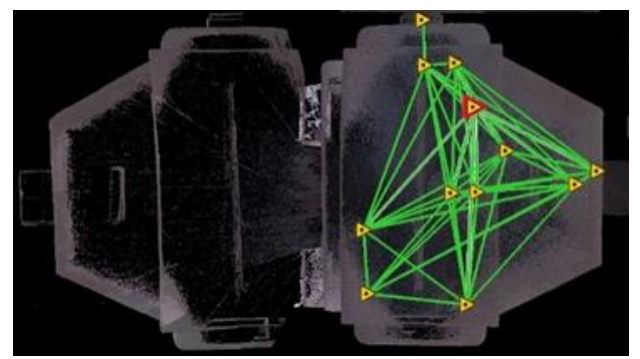

Fig. 11. The 3D inverse image

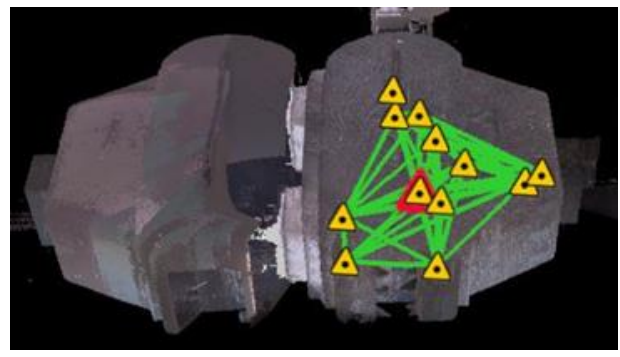

Fig. 12. The 3D model in the structure of a point cloud

\section{Conclusion}

An experiment deals with the influence of the environment and lighting on the image quality of the scanned area using the 3D scanner confirmed the influence of the white area on the degradation of the image by noise, especially in areas with shadows. This affected the information about the image at individual points in the point cloud. To increase the image quality, the basic brightness and contrast values have been increased by $+90 \%$. The goal was to suppress noise. Brightness as an image value and contrast are essential tools for improving image quality.

These values can be adjusted on the devices before the image is taken. Here, however, the input values have been retained. In the scanned environment, it was proven that obtain higher image quality only by changing one of the values. But not much. By changing both values at the same time, on the contrary, the image quality was more degraded. The interior mirror affected the image quality by reflecting the laser beam as well. The inverse 3D model of the interior was created. Inverse images and dots describing information in the point cloud can provide the solution to increasing image quality directly in the 3D model of the point cloud.

Occlusion of the point clouds from both 3D models in areas with low image quality and reflected shadow on a white surface. The occlusion of some selected areas in the point cloud of the inverse image is linked to this experiment to increase the image quality directly in the 3D model of the scanned space.

\section{Acknowledgments}

This research was based on the support of the Internal Grant Agency of Tomas Bata University under project No. IGA/CebiaTech/2020/003.

\section{References}

[1] Vijayalakshmi, D.; Malaya K. N.; Acharya P. O. (2020). A Comprehensive Survey on Image Contrast Enhancement Techniques in Spatial Domain, Sensing and Imaging, Vol. 21, No. 1, 2020, DOI: 10.1007/s11220-020-00305-3

[2] Altuntas, C., (2015). Integration of point clouds originated prom laser and photogrammetric images for visualition of complex details of historical buildings, Int. Arch. Photogramm. Remote Sens. Spatial Inf. Sci., XL-5/W4, 431435, 2015, DOI: https://doi.org/10.5194/isprsarchives-XL-5-W4-431-2015

[3] Hlaváč, V. \& Šonka, M. (1992), Počítačové vidění, Grada a.s., ISBN: 80-85424-67-3

[4] Griffiths, D.; Boehm, J., (2019). A Review on Deep Learning Techniques for 3D Sensed Data Classification, Remute Sensing, Vol. 11, No. 12, ISSN: 2072-4292, DOI: https://doi.org/10.3390/rs11121499

[5] Agisoft (2020). [online], https://www.agisoft.com/features/professional-edition/

[6] Gefos ČR Leica BLK360 (2020). [online], https://www.gefos-leica.cz/o-produktech/3d-laserove-skenovani/3d-laserove-skenery/blk360

[7] Gefos ČR Leika BLK360 User Manual (2020). [online], https://www.gefos-leica.cz/data/original/skenery/rtc/853811_leica_blk360_um_v2.0.0_en.pdf

[8] Švejda, J. (2018) Testing of the properties of the Leica ScanStation P40 and BLK360 3D scanners, Thesis, CVUT in Praque, 2018. 